\title{
The Role of the Dorsal Hippocampus in the Acquisition and Retrieval of Context Memory Representations
}

\author{
Patricia Matus-Amat, Emily A. Higgins, Ruth M. Barrientos, and Jerry W. Rudy \\ Department of Psychology, Center for Neuroscience, University of Colorado, Boulder, Colorado 80309
}

It is argued that the hippocampus contributes to contextual fear conditioning by supporting the acquisition of a conjunctive memory representation of context, which associates with shock. This function was examined by studying the context pre-exposure facilitation effect (CPFE). A rat that is shocked immediately after being placed into a context subsequently displays almost no fear of that context. However, if it is pre-exposed to the context the day before immediate shock, it displays significant freezing to that context. By using 5-aminomethyl-3-hydroxysoxazole to temporarily inactivate the dorsal hippocampus $(\mathrm{DH})$ at three different phases of the procedure, which produces the CPFE, we show that the hippocampus is necessary for the following: (1) acquisition of the context memory, (2) retrieval of this memory at the time of immediate shock, and (3) retrieval of the context-shock memory at the time of testing. In contrast, inactivating the DH before a standard contextual shock experience had no effect on contextual fear conditioning. These results support the view that two processes can support contextual fear conditioning: (1) conditioning to the conjunctive representation, which depends on the hippocampus, and (2) conditioning to the features that make up the context, which does not.

Key words: hippocampus; contextual fear conditioning; context pre-exposure facilitation effect; conjunctive representation; pattern completion; declarative memory

\section{Introduction}

Although it is often claimed that contextual fear conditioning depends on the hippocampus, the evidence is mixed (Gewirtz et al., 2000; Sanders et al., 2003). Retrograde damage to the hippocampus impairs the retention of contextual fear conditioning (Kim and Fanselow, 1992; Maren et al., 1997; Frankland et al., 1998; Anagnostaras et al., 1999). However, the pattern of data associated with anterograde damage is complex. Electrolytic damage to the dorsal hippocampus (DH) and excitotoxic damage to the ventral hippocampus each impairs conditioning (Selden et al., 1991; Phillips and LeDoux, 1992, 1994; Maren and Fanselow, 1998), but excitotoxic damage to the DH has no effect (Maren et al., 1997; Richmond et al., 1999; Rudy et al., 2002). There is some agreement that the anterograde impairment is a product of hyperactivity, which is a consequence of damaging a circuit that includes cells in the ventral hippocampus and fibers that transverse through the $\mathrm{DH}$ to the nucleus accumbens (Maren et al., 1997; Richmond et al., 1999). Excitotoxic damage to the DH does not produce impairment, because the fibers of passages are spared and no hyperactivity is produced. Consequently, the literature indicates that the acquisition of contextual fear does not require the $\mathrm{DH}$.

Some theorists propose that the mixed anterograde and retrograde results mean that contextual fear conditioning can be

\footnotetext{
Received July 2, 2003; revised Jan. 8, 2004; accepted Jan. 8, 2004.

This work was supported by National Institutes of Health Grant R01 MH613164.

Correspondence should be addressed to Jerry W. Rudy, 345 UCB, Department of Psychology, University of Colorado, Boulder, C0 80309.E-mail: jrudy@psych.colorado.edu.

DOI:10.1523/JNEUROSCI.1598-03.2004

Copyright $\odot 2004$ Society for Neuroscience $\quad 0270-6474 / 04 / 242431-09 \$ 15.00 / 0$
}

supported by two processes: (1) the independent features that make up the context and are associated with shock, which does not require the hippocampus, or (2) the hippocampus binding these features into a conjunctive representation that associates with shock (Fanselow, 1999, 2000; Rudy and O'Reilly, 1999, 2001). The feature-shock associations support contextual fear in rats with anterograde damage (Maren et al., 1997; Frankland et al., 1998; Rudy et al., 2002). Retrograde damage impairs contextual fear conditioning, because in the intact rat, conditioning to the conjunctive representation overshadows conditioning to the features, and this representation is lost when the hippocampus is subsequently damaged (Maren et al., 1997).

The purpose of these experiments is to provide evidence that the hippocampus supports a conjunctive representation of context. The experiments focus on the context pre-exposure facilitation effect (CPFE). If a rat is placed into a context and shocked immediately, it will display almost no fear of that context. However, if it is pre-exposed to the context the day before it is immediately shocked, it will display substantial freezing (Fanselow, 1990). We provided evidence that context pre-exposure facilitates contextual fear conditioning because the rat acquires a conjunctive representation of the context (Rudy and O'Reilly, 1999, 2001).

The CPFE requires a three-stage training procedure: (1) preexposure to the context, (2) immediate shock, and (3) the test for context fear. According to the conjunctive theory, the hippocampus makes a significant contribution to each stage of training. To examine the contribution that the hippocampus makes to each stage of the experiment, we used 5-aminomethyl-3hydroxysoxazole (muscimol), a $\mathrm{GABA}_{\mathrm{A}}$ agonist that potentiates inhibitory synaptic transmission in the brain (Martin and Ghez, 1999; Edeline et al., 2002) to temporarily inactivate the DH. 


\section{Materials and Methods Subjects}

Adult male Long-Evans rats weighing 250-300 gm at the start of the experiments were bred at the University of Colorado. Rats were housed in groups of three to four in plastic cages with ad libitum access to food and water, maintained on a $12 \mathrm{hr}$ light/dark cycle, and given 1 week to acclimate to colony conditions before experimentation began. All experiments were conducted in accordance with protocols approved by the University of Colorado Animal Care and Use Committee.

\section{Surgery}

Under halothane anesthesia, animals were placed into a stereotaxic apparatus (David Kopf Instruments, Tujunga, CA), and stainless-steel guide cannulas (Plastics One, Roanoke, VA) were implanted bilaterally into the DH (Plastics One). Based on the rat brain atlas of Paxinos and Watson (1997), the following coordinates were used for bilateral DH implantation: anteroposterior (AP), $-3.5 \mathrm{~mm}$; mediolateral (ML), \pm 2.4 $\mathrm{mm}$; dorsoventral (DV), $-3.0 \mathrm{~mm}$ relative to bregma. In experiment $2 \mathrm{a}$, cannulas were implanted bilaterally into the parietal cortex (area 1) just lateral to the $\mathrm{DH}(\mathrm{AP},-3.5 \mathrm{~mm}$; ML, $\pm 5.5 \mathrm{~mm}$; DV,$-3.0 \mathrm{~mm}$ relative to bregma). In experiment $2 \mathrm{~b}$, a cannula was placed into the lateral ventricle (counterbalancing for hemisphere). The coordinates were as follows: AP, $-0.92 \mathrm{~mm}$; ML, $\pm 1.5 \mathrm{~mm}$; DV,$-3.5 \mathrm{~mm}$. Cannulas were fixed to the skull with dental acrylic and three small screws. To maintain patency, after surgery, an obturator was placed into the guide cannulas that extended $1 \mathrm{~mm}$ beyond the tip of the guide cannula (total length, 4 $\mathrm{mm})$. Rats were allowed to recover for $7-10 \mathrm{~d}$ before being trained on the behavioral task.

\section{Apparatus}

Pre-exposure, shock, and testing were conducted in two identical ice chests $(54 \times 30 \times 27 \mathrm{~cm})$ with white interiors. The conditioning chambers $(26 \times 21 \times 24 \mathrm{~cm})$, placed inside each chest, were made of clear plastic and had window screen tops. A speaker and an activated $120 \mathrm{~V} \mathrm{AC}$ $6 \mathrm{~W}$ light bulb were mounted on the ceiling of each chest. The $2 \mathrm{sec}, 1.5$ $\mathrm{mA}$ shock was delivered through a removable floor with stainless-steel rods $5 \mathrm{~mm}$ in diameter, spaced $1.65 \mathrm{~cm}$ center to center. Each rod was wired to a shock generator and scrambler (Coulbourn Instruments, Allentown, PA). Each chamber was cleaned with water before each animal was pre-exposed, shocked, or tested.

\section{Behavioral procedures}

Rats were taken two at a time from their home cage and transported to the conditioning context in a black ice bucket. The lid was on so that the rats could not see where they were being taken. Rats were placed in the context, allowed to explore freely for $5 \mathrm{~min}$, and then transported back to their home cage where they remained $\sim 40 \mathrm{sec}$ before the next exposure. During the five subsequent exposures, the animals remained in the context for $\sim 40 \mathrm{sec}$ and then were returned to their home cage for $40 \mathrm{sec}$. The purpose of the multiple exposures was to establish the features of the black bucket as retrieval cues that could activate the representation of the context. Twenty-four hours later, the rats were taken from their home cage and transported individually to the conditioning context in the black bucket. There they received a single 2 sec shock immediately after being placed in the context. They were immediately removed and transported back to their home cage. Contextual fear was assessed $24 \mathrm{hr}$ after immediate shock by placing the rat in the conditioning context for $6 \mathrm{~min}$. Every $10 \mathrm{sec}$, each rat was judged as either freezing or active at the instant the sample was taken. Freezing, the rat's dominant defensive fear response, is a complete suppression of behavior that is accompanied by immobility, shallow breathing, and a variety of other autonomic changes, including an increase in heart rate and piloerection. Freezing in these experiments was defined as the absence of all visible movement, except for respiration. Scoring began $\sim 10 \mathrm{sec}$ after the animal was placed into the chamber. The scorers were blind to experimental treatment, and interscorer reliability was $>95 \%$ for all experiments.

\section{Microinjections}

Depending on the experiment, microinjections were administered at either $1 \mathrm{hr}$ before training or immediately after training. Rats were gently wrapped in a soft towel, the obturator was removed, and a 33 gauge microinjector (Plastics One) attached to polyethylene 50 (PE50) tubing was inserted through the indwelling guide cannula. The distal end of the PE50 tubing was attached to a $100 \mu \mathrm{l}$ (Hamilton, Reno, NV) syringe that was attached to a microinjection unit (model 5000; David Kopf Instruments) that accurately dispensed the desired volume, which was injected over $10-15 \mathrm{sec}$. For experiments using bilateral intrahippocampal injections, $0.5 \mu \mathrm{l}$ was injected sequentially into each hemisphere. The microinjector remained in place for $2 \mathrm{~min}$. Muscimol, a GABA agonist provided by Sigma (St. Louis, MO), was microinjected in the $\mathrm{DH}$ at a concentration of $1 \mu \mathrm{g} / \mu \mathrm{l}, 0.5 \mu \mathrm{l}$ in each side. This dose has been successfully used in other recent experiments to inactivate the $\mathrm{DH}$ (Holt and Maren, 1999a; Corcoran and Maren, 2001). Vehicle controls received equivolume sterile PBS, $\mathrm{pH}$ 7.4. In experiment 2 , the drug and vehicle were injected intracerebroventricularly. During surgery, a standard drip procedure was used to verify the cannula placement (Watkins et al., 1992).

\section{Histology}

At the completion of the experiment, animals were anesthetized with Nembutal $(50 \mathrm{mg} / \mathrm{kg})$ and decapitated; their brains were removed and frozen in cold isopentane. Coronal sections ( $40 \mu \mathrm{m}$ thick) were taken through the hippocampus with a cryostat at $-19^{\circ} \mathrm{C}$, and every third section was mounted. Sections were stained with cresyl violet and examined by light microscopy to visually verify the placement of the cannula in the dorsal hippocampus. Only rats with proper cannula placements were included in the analyses of each experiment.

\section{Results}

\section{Experiment 1: muscimol injected into dorsal hippocampus before context pre-exposure prevents acquiring a representation of context}

The purpose of the first experiment was to determine whether inactivating the $\mathrm{DH}$ before context exposure would prevent learning about the context. Before pre-exposure, muscimol or vehicle was injected bilaterally into the $\mathrm{DH}$. The next day, the rats were brought to the conditioning room, placed in the conditioning context, and immediately shocked. All animals were tested 24 hr later. As noted, immediate shock normally produces very little contextual fear conditioning, because there is no representation of context available to associate with shock. Pre-exposure to the context allows the rat to acquire a representation of context and enhances conditioning normally produced by immediate shock. Thus, rats pre-exposed to the context and treated with the vehicle should show strong fear conditioning. However, rats injected with muscimol should display much less fear conditioning, because inactivating the $\mathrm{DH}$ should prevent the rat from acquiring the representation of context.

The injection cannula tip placements for all animals included in the analysis of this experiment are shown in the top panel of Figure 1. There were nine subjects in each condition. Cannula placements were localized throughout the rostrocaudal extent of the DH. Cannula placements for vehicle- and muscimol-injected animals were basically the same; thus any difference between the two conditions was not a product of differential cannula placement.

The bottom panel of Figure 1 shows that rats injected with the vehicle before context pre-exposure displayed substantial freezing. In contrast, rats injected with muscimol displayed markedly reduced freezing $\left(F_{(1,16)}=6.85 ; p<0.02\right.$. $)$ These results suggest that inactivating neurons in the $\mathrm{DH}$ with muscimol prevented rats from acquiring a representation of the context. However, it is 

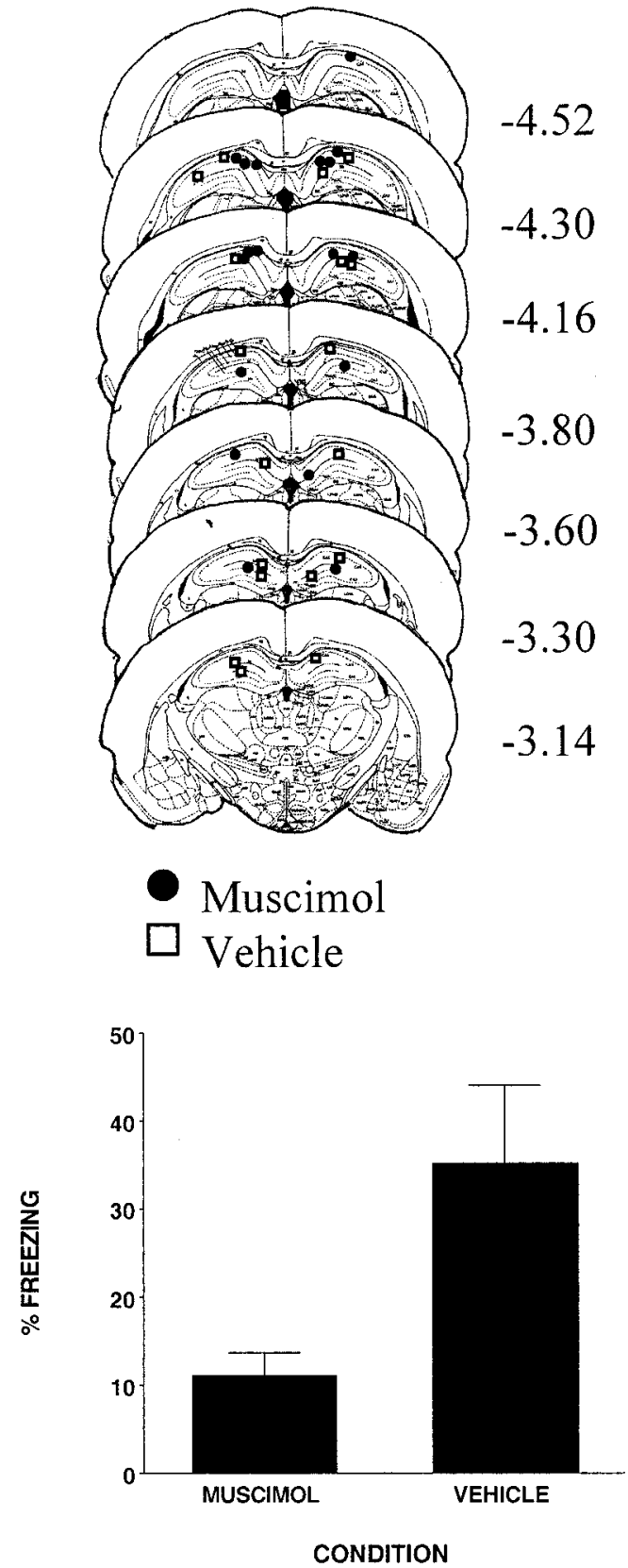

Figure 1. Top, Schematic representation of injection cannula tip placements in the dorsal hippocampus for all rats included in experiment 1 . The values to the right of each coronal section indicate the position of each section relative to bregma. Coronal brain images are adapted from the atlas of Paxinos and Watson (1997). Bottom, Muscimol infused into the dorsal hippocampus $1 \mathrm{hr}$ before context pre-exposure significantly reduced freezing resulting from immediate shock. Error bars indicate mean \pm SEM percentage of freezing during the context fear test.

possible that the effect of muscimol was a result of it diffusing beyond the intended sites in the DH (Martin, 1991; Edeline et al., 2002).

Experiments $2 \mathrm{a}$ and $2 \mathrm{~b}$ : is the effect of muscimol caused by diffusion beyond the dorsal hippocampus?

We conducted two experiments to determine whether the results of experiment 1 were attributable to muscimol diffusing beyond the DH. In experiment $2 \mathrm{a}$, the same dose of muscimol $(n=7)$ or vehicle $(n=7)$ was injected bilaterally into the parietal cortex (area 1 just lateral to the $\mathrm{DH}$ ). In experiment 2b, the same dose of muscimol $(n=8)$ or vehicle $(n=7)$ used in experiment 1 was injected intracerebroventricularly.

The injection cannula tip placements for all animals included in the analysis of experiment $2 \mathrm{a}$ are shown in the top panel of Figure 2. It shows that the cannula placements for vehicle- and muscimol-injected animals were in the intended cortical sites.

The behavioral results for experiments $2 \mathrm{a}$ and $2 \mathrm{~b}$ are shown in the bottom two panels of Figure 2. The results indicate that injecting muscimol before context pre-exposure had no effect ( $F$ values of $<1$ ) on the CPFE, whether it was injected into the cortex just lateral to the $\mathrm{DH}$ or intracerebroventricularly. These results provide no evidence that the effect of muscimol in experiment 1 was the result of it diffusing into sites beyond the hippocampus.

Experiment 3: muscimol after context pre-exposure has no effect on the acquisition of a context representation It is also possible that the results of experiment 1 were attributable to some muscimol-induced permanent damage to the $\mathrm{DH}$, which could have influenced test performance. To evaluate this possibility, rats were pre-exposed to the context. Immediately after pre-exposure, either muscimol or vehicle was injected into the $\mathrm{DH}$. If the results of experiment 1 were a result of some permanent alteration of hippocampal function, then injecting muscimol after pre-exposure should also impair test performance.

The injection cannula tip placements for all animals included in the analysis of this experiment are shown in the top panel of Figure 3. There were eight subjects in each condition. As in experiment 1 , cannula placements were localized throughout the rostrocaudal extent of the DH. Cannula placements for vehicleand muscimol-injected animals were basically the same; thus any difference between the two conditions was not a product of differential cannula placement.

The bottom panel of Figure 3 shows that rats injected with muscimol immediately after context pre-exposure did not differ $(F<1)$ from rats that were treated with the vehicle. Thus, the results of experiment 1 cannot be attributed to any residual effect of muscimol on hippocampal function that carried over to influence either the conditioning or the test phase of the experiment. They also indicate that inactivating the DH immediately after pre-exposure did not interfere with the consolidation of the memory for context. Thus, together with experiment 1 , these results support the hypothesis that muscimol injected into the $\mathrm{DH}$ before context pre-exposure prevents rats from learning about the context.

\section{Experiment 4: muscimol before conditioning does not affect} normal contextual fear conditioning

We provided evidence that the normal rat acquires a conjunctive representation when it is allowed to explore the context (Rudy and O'Reilly, 1999, 2001). Thus, it is likely that in experiment 1 , muscimol prevented rats from acquiring the conjunctive representation during the pre-exposure phase of the experiment. In experiment 4 , the effect of muscimol on normal fear conditioning was examined. This experiment is interesting on both empirical and theoretical grounds. Recall that excitotoxic, anterograde damage to the DH does not impair normal contextual fear conditioning (Maren et al., 1997; Richmond et al., 1999; Rudy et al., 2002). It is possible that inactivating the $\mathrm{DH}$ might be functionally equivalent to an excitotoxic lesion and also not influence contextual fear conditioning. On theoretical grounds, this result is possible because conditioning to the features of the context, which does not require the hippocampus, can support contextual fear conditioning (Fanselow, 2000; Rudy and O'Reilly, 2001; 

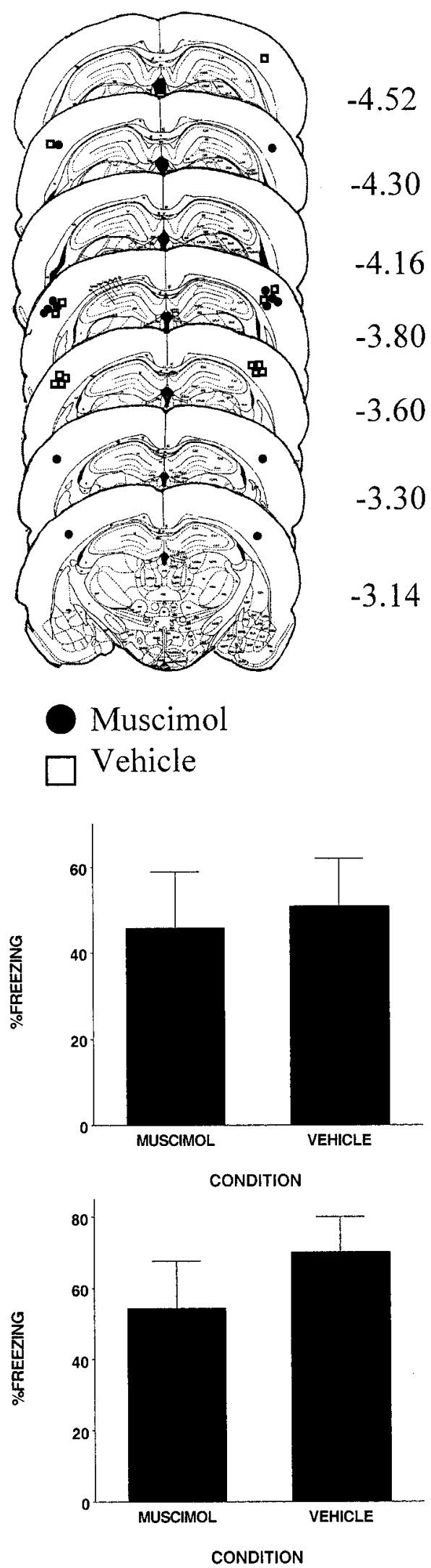

Figure 2. Top, Schematic representation of injection cannula tip placements for all rats included in experiment 2a. The values to the right of each coronal section indicate the position of each section relative to bregma. Coronal brain images are adapted from the atlas of Paxinos and Watson (1997). Bottom, Muscimol infused intracerebroventricularly or into the parietal cortex $1 \mathrm{hr}$ before context pre-exposure did not affect freezing resulting from immediate shock (experiment 2). Error bars indicate mean \pm SEM percentage of freezing during the context fear test.

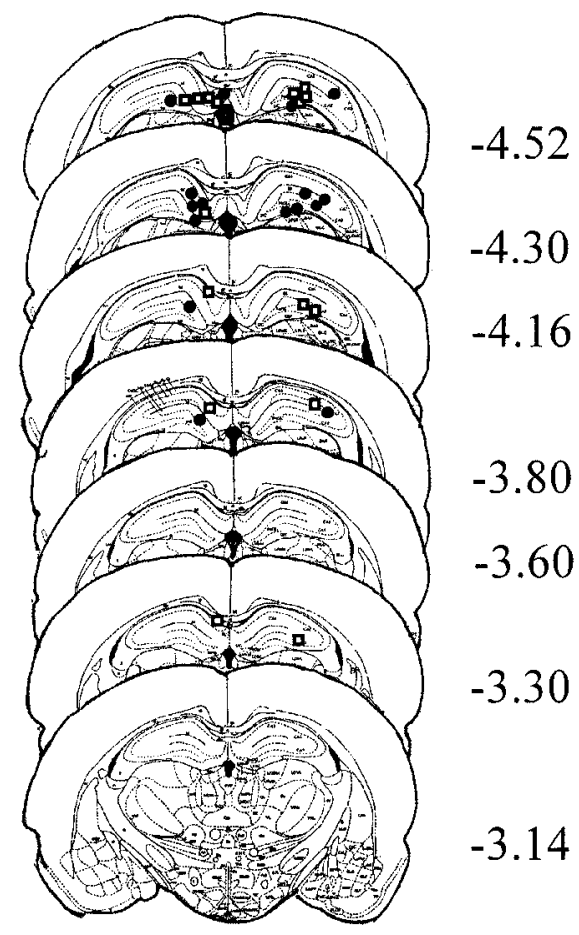

Muscimol
$\square$ Vehicle

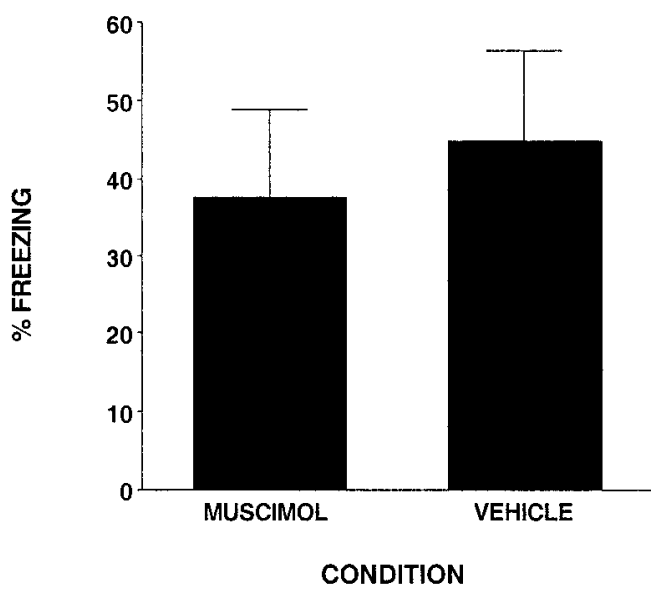

Figure 3. Top, Schematic representation of injection cannula tip placements in the dorsal hippocampus for all rats included in experiment 3 . The values to the right of each coronal section indicate the position of each section relative to bregma. Coronal brain images are adapted from the atlas of Paxinos and Watson (1997). Bottom, Muscimol infused into the dorsal hippocampus immediately after context pre-exposure did not affect freezing resulting from immediate shock. Error bars indicate mean \pm SEM percentage of freezing during the context fear test.

Rudy et al., 2004). To assess this possibility, rats were given a standard single-shock fear conditioning experience. Two minutes after being placed into the conditioning chamber, the rat received a 2 sec shock. Before conditioning, either muscimol or vehicle was injected into the $\mathrm{DH}$. Two independent replications of this experiment were conducted.

The injection cannula tip placements for all animals included in the analysis of the two replications of this experiment are shown in the top panel of Figure 4 . There were 16 subjects in each condition. As in the other experiments, cannula placements were 

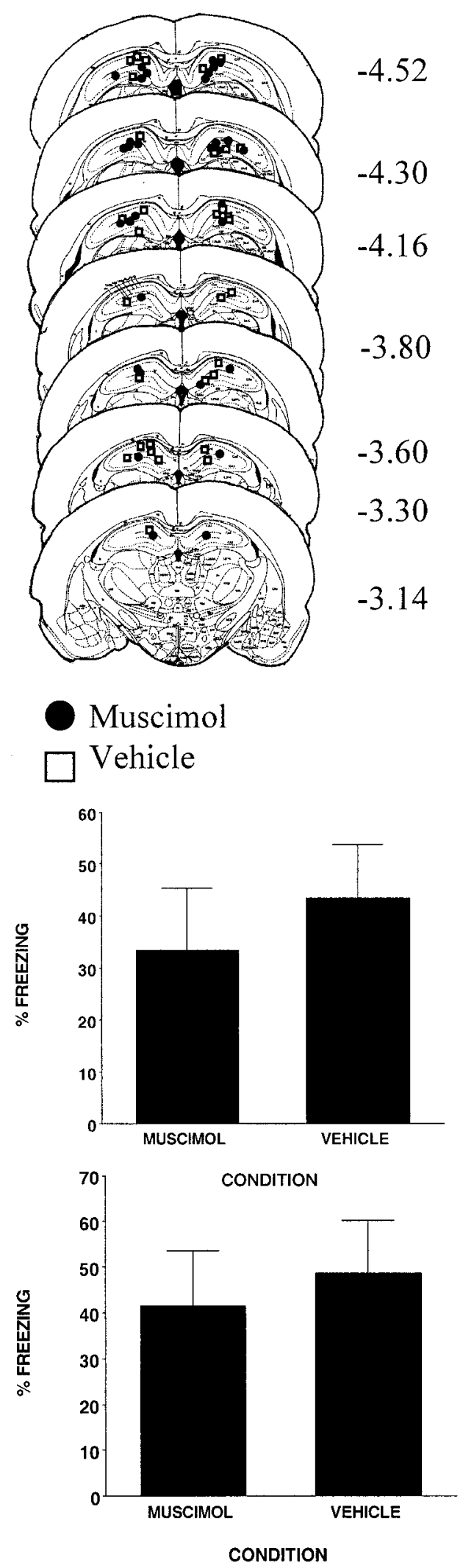

Figure 4. Top, Schematic representation of injection cannula tip placements in the dorsal hippocampus for all rats included in experiment 4 . The values to the right of each coronal section indicate the position of each section relative to bregma. Coronal brain images are adapted from the atlas of Paxinos and Watson (1997). Bottom, Muscimol infused into the dorsal hippocampus $1 \mathrm{hr}$ before a standard contextual fear conditioning experiment did not affect freezing. There were two independent replications of this experiment. Error bars indicate mean \pm SEM percentage of freezing during the context fear test. localized throughout the rostrocaudal extent of the DH. Cannula placements for vehicle- and muscimol-injected animals were basically the same; thus any difference between the two conditions was not a product of differential cannula placement.

The bottom two panels of Figure 4 show the results of the two replications of this experiment. It reveals that muscimol given before a standard contextual fear conditioning experience had no effect $(F$ values of $<1)$. These results are in contrast to the results of experiment 1 , in which the same dose of muscimol blocked the effect of context pre-exposure. However, they are consistent with studies that have shown that anterograde excitotoxic damage to the hippocampus does not impair contextual fear conditioning (Maren et al., 1997; Richmond et al., 1999; Rudy et al., 2002). Thus, inactivating the hippocampus with muscimol appears to be functionally similar to an excitotoxic DH lesion. The combined results of experiments 1 and 4 are consistent with the hypothesis that contextual fear conditioning can be supported by two processes: conditioning to the conjunctive representation, which depends on the hippocampus (experiment 1), and conditioning to context features, which does not depend on the hippocampus (experiment 2).

That previous treatment with muscimol had no effect on standard contextual fear conditioning is also important, because it indicates that muscimol did not disrupt the normal pattern of exploratory behavior needed to sample the features of the context or to condition to them. However, we note that this statement is an inference we make from the fact that the rats conditioned, and we did not measure the exploratory behavior of the rats.

However, it should be noted that our results conflict with those reported by Bellgowan and Helmstetter (1995). We have no ready explanation of the conflicting results, but note that, consistent with our results, Holt and Maren (1999b) also failed to find an effect of muscimol on the acquisition of contextual fear conditioning.

\section{Experiment 5: retrieval of the conjunctive representation before immediate shock depends on the hippocampus} As noted, the immediate-shock experiment is composed of three distinct phases: (1) pre-exposure to the context, (2) immediate shock, and (3) the test for context fear. Experiment 1 demonstrated that inactivating the $\mathrm{DH}$ prevented the acquisition of the conjunctive context representation. It is important to appreciate that for this representation to facilitate conditioning produced by immediate shock, it has to be retrieved and available to be associated with the immediate shock. Indeed, we reported previously that immediately shocked rats must be conditioning to the memory representation of where they were pre-exposed and not to physical features of the place where they experienced immediate shock (Rudy and O'Reilly, 2001; Rudy et al., 2002). How does this happen?

One of the important properties of hippocampal-dependent conjunctive representations is that they support what is called pattern completion (Marr, 1971; McNaughton and Morris, 1987; Rudy and O'Reilly, 1999, 2001; O'Reilly and Rudy, 2001). Specifically, when an input pattern of features (A-E) is conjoined into a memory representation, the presentation of a subset of features (e.g., A and E) will activate the memory for the entire input pattern. It is the pattern-completing properties of the hippocampus that make it possible for immediate shock to support conditioning to the pre-exposed context. By virtue of the pre-exposure experience, the rat acquires a conjunctive representation of the context together with the cues associated with transporting the rat to the context. Consequently, on the day of immediate shock, 
the transport cues activate the memory representation of the context so that it is available to associate with immediate shock (Rudy and O'Reilly, 2001; Rudy et al., 2002).

This line of reasoning makes an unambiguous prediction. Specifically, because pattern completion (1) is needed to activate the memory representation of the pre-exposed context at the time of immediate shock and (2) requires a hippocampaldependent conjunctive representation, it predicts that inactivating the hippocampus before immediate shock will prevent the context pre-exposure facilitation effect. To test this prediction, all rats were pre-exposed to the conditioning context in the normal state, and either muscimol or vehicle was injected bilaterally into the $\mathrm{DH}$ before the immediate shock session.

The injection cannula tip placements for all animals included in the analysis of this experiment are shown in the top panel of Figure 5. There were six subjects in each condition. Cannula placements were localized throughout the rostrocaudal extent of the DH. Cannula placements for vehicle- and muscimol-injected animals were basically the same; thus any differences between the two conditions were not a product of differential cannula placement.

The bottom panel of Figure 5 shows that rats injected with muscimol before immediate shock display much less contextual fear than rats injected with the vehicle $\left(F_{(1,10)}=21.8 ; p<0.001\right)$. This result strongly supports the view that the patterncompletion process, which is needed to activate the memory representation of the context at the time of immediate shock, depends on a conjunctive representation supported by the hippocampus.

There is much literature centered on the contribution the hippocampus makes to the acquisition and consolidation of memories. Yet very few studies have specifically addressed the role of the hippocampus in memory retrieval. However, Maren and colleagues (Corcoran and Maren, 1999a; Holt and Maren, 2001) recently used the muscimol inactivation strategy to show that the hippocampus is critically involved in two other phenomena that involve context representations. Specifically, they have reported that the hippocampus makes an essential contribution to the retrieval of context-specific latent inhibition (Holt and Maren, 1999a) and context-specific extinction (Corcoran and Maren, 2001). These results are thus consistent with those of Maren and colleagues (Corcoran and Maren, 1999a; Holt and Maren, 2001) in implicating an important role for the hippocampus in retrieving contextual information.

\section{Experiment 6: expression of fear is impaired by} intrahippocampal injections of muscimol

The previous experiments have demonstrated that if the context pre-exposure facilitation effect is to be observed, the hippocampus must be functional both during the initial pre-exposure phase and at the time of immediate shock, when the representation of the context must be retrieved to associate with shock. Because the context pre-exposure facilitation effect is mediated by the conjunctive representation of context, then it should also be the case that inactivating the hippocampus should impair the rat's performance during the test for contextual fear. To evaluate this prediction, all rats were in the normal state both at the time of pre-exposure to the context and during the immediate shock phase. However, before the test for contextual fear, rats received an injection of muscimol or vehicle.

The injection cannula tip placements for all animals included in the analysis of this experiment are shown in the top panel of Figure 6. There were seven subjects in each condition. Cannula placements were localized throughout the rostrocaudal extent of the
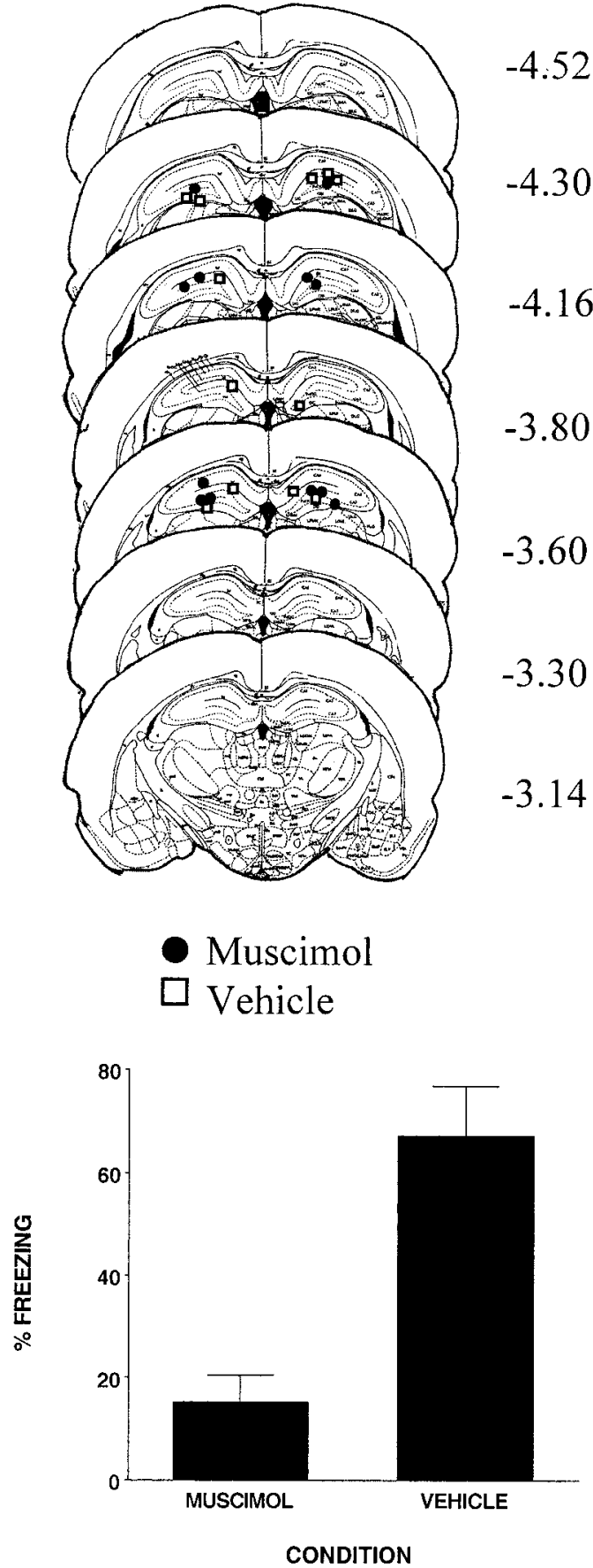

Figure 5. Top, Schematic representation of injection cannula tip placements in the dorsal hippocampus for all rats included in experiment 5 . The values to the right of each coronal section indicate the position of each section relative to bregma. Coronal brain images are adapted from the atlas of Paxinos and Watson (1997). Bottom, Muscimol infused into the dorsal hippocampus $1 \mathrm{hr}$ before immediate shock significantly reduced freezing resulting from immediate shock. Error bars indicate mean \pm SEM percentage of freezing during the context fear test.

DH. Cannula placements for vehicle- and muscimol-injected animals were basically the same; thus any difference between the two conditions was not a product of differential cannula placement.

The bottom panel of Figure 6 shows that rats injected with muscimol before the test for contextual fear displayed reduced freezing compared with rats that were injected with the vehicle $\left(F_{(1,12)}=6.3 ; p<0.03\right)$. We argued that during the immediate shock phase, rats associated a hippocampal-dependent conjunc- 

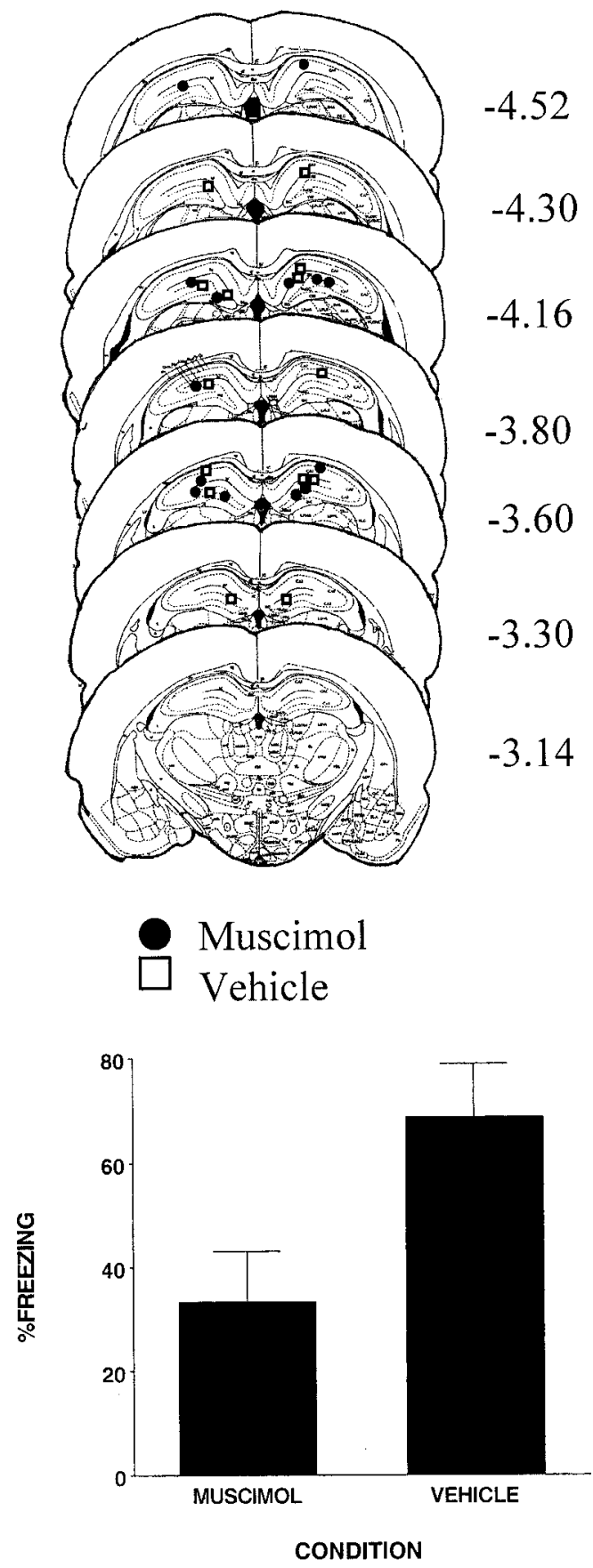

Figure 6. Top, Schematic representation of injection cannula tip placements in the dorsal hippocampus for all rats included in experiment 6 . The values to the right of each coronal section indicate the position of each section relative to bregma. Coronal brain images are adapted from the atlas of Paxinos and Watson (1997). Bottom, Muscimol infused into the dorsal hippocampus $1 \mathrm{hr}$ before the test for contextual fear significantly reduced freezing. Error bars indicate mean \pm SEM percentage of freezing during the context fear test.

tive representation of context with the shock. If this is true, then for fear to be displayed during testing, the hippocampus is needed to retrieve this representation. Consequently, when the hippocampus is inactivated, rats should display reduced freezing. The results of this experiment are consistent with this prediction.

Experiment 7: the effects of muscimol are not state dependent The results of these experiments all point to a role for the hippocampus in storing and retrieving a conjunctive memory of the
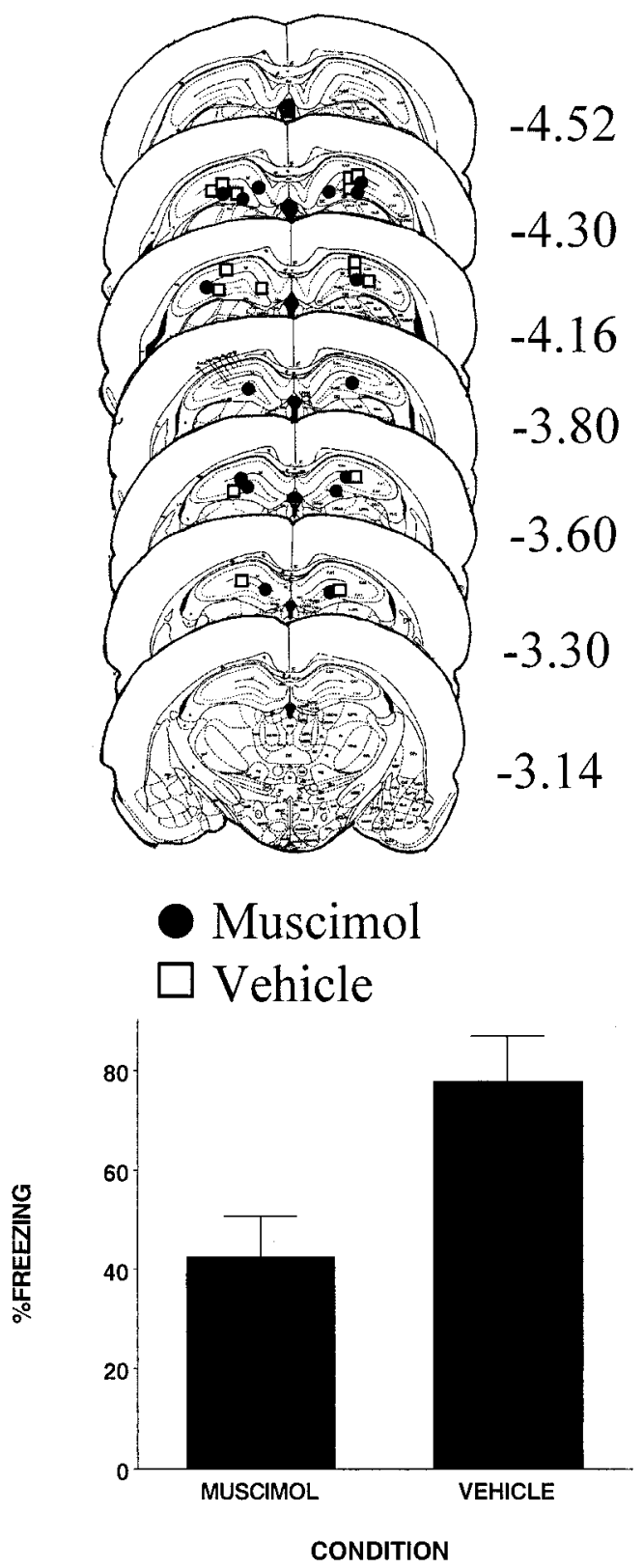

Figure 7. Top, Schematic representation of injection cannula tip placements in the dorsal hippocampus for all rats included in experiment 7 . The values to the right of each coronal section indicate the position of each section relative to bregma. Coronal brain images are adapted from the atlas of Paxinos and Watson (1997). Bottom, Muscimol infused into the dorsal hippocampus $1 \mathrm{hr}$ before each phase of the experiment significantly reduced freezing. Error bars indicate mean \pm SEM percentage of freezing during the context fear test.

context. However, all of these findings can potentially be explained as reflecting what is called state-dependent learning (for review, see Overton, 1991). A fundamental principle of memory is that the retrieval of a memory depends critically on the similarity of the cues present during the acquisition of the memory to the cues present at the time the memory is retrieved (Spear, 1973). To the degree that training and retrieval cues differ, memory retrieval will be impaired. Moreover, it has been shown that the cues produced by the internal state of the subject at the time of learning can become part of the memory trace (Overton, 1991). Thus, if the subject's state during learning differs from its state at the time of testing, memory retrieval should be impaired. State variables include factors such as circadian rhythm, mood, and drug-induced 
state. The state-dependent learning hypothesis provides a potential explanation of our primary findings. In the first experiment, the memory trace representing the phase 1 context pre-exposure experience could consist not only of the representation of the physical features of the context but also of an internal state of the hippocampus produced by muscimol. Consequently, because these animals were not treated with muscimol either during the phase 2 retrieval of the memory or during the phase 3 test for contextual fear, the retrieval cues may not match the training cues, and impaired performance would result from this difference.

To address the state-dependent learning hypothesis, muscimol was injected into the DH before each of the three phases of the experiment (pre-exposure, immediate shock, and testing) for one group of animals, and vehicle was injected into the other group of animals. To ensure that repeated injections of muscimol into the $\mathrm{DH}$ did not produce any behavioral impairment, a $5 \mathrm{~d}$ interval separated each phase of the experiment.

The injection cannula tip placements for all animals included in the analysis of this experiment are shown in the top panel of Figure 7. There were eight subjects in the muscimol condition and six in the vehicle condition. Cannula placements were localized throughout the rostrocaudal extent of the DH. Cannula placements for vehicle- and muscimol-injected animals were basically the same; thus any difference between the two conditions was not a product of differential cannula placement.

The bottom panel of Figure 7 shows that rats injected with muscimol before each phase of the experiment displayed reduced freezing compared with rats that were injected with the vehicle $\left(F_{(1,12)}=8.3 ; p<0.02\right)$. Injecting the rats with muscimol before each stage of the experiment should have ensured that state cues generated by muscimol would be the same during the acquisition and retrieval stages of the experiment. Therefore, the statedependent learning hypothesis would predict that these rats should not differ from the rats injected with the vehicle. However, even after controlling for the state of the rat, a deficit was still observed. Thus, it is fair to conclude that these results provide no evidence that the influence of muscimol on the CPFE is attributable to its effect on the state of the rat.

\section{Discussion}

Muscimol injected into the DH before (1) context pre-exposure, (2) immediate shock, or (3) the test for contextual fear conditioning significantly reduced the CPFE. There was no evidence that these results were attributable to the muscimol diffusing beyond its intended sites. Thus, these findings are consistent with the hypothesis that neurons in the DH make a significant contribution to the acquisition of the memory for context, its retrieval at the time of testing, and its retrieval at the time of the fear test.

Although injecting muscimol into the DH before context exposure interfered with the acquisition of the memory for context (as indicated by the reduced CPFE), it did not impair the acquisition of contextual fear conditioning when it was injected before standard conditioning (experiment 4). The two-process theory of contextual fear conditioning (Fanselow, 2000; O'Reilly and Rudy, 2001; Rudy et al., 2004) readily explains these somewhat paradoxical results. According to this view, contextual fear conditioning can be produced by conditioning either to the conjunctive representation of the context, which depends on the hippocampus, or to the independent features of the context, which does not. Consequently, although muscimol inactivated the $\mathrm{DH}$, freezing was robust because there was conditioning to the features. In contrast, the CPFE depends uniquely on the rat acquiring the hippocampus-dependent conjunctive representation
(Rudy and O'Reilly, 1999), so injecting muscimol into the DH before pre-exposure impairs this effect. To understand this claim, it is necessary to appreciate that freezing, which is observed in the test phase of the CPFE paradigm, is produced by immediate shock. Because shock is immediate, the rat does not have the opportunity to sample and encode the features of the shock context. In fact, if the rat is not pre-exposed to the context, immediate shock will produce almost no conditioning (Fanselow, 1990; Kiernan and Westbrook, 1993). This means that the pre-exposed rat must condition to the retrieved conjunctive memory of the context and not to its sensory features. We provided strong evidence for this analysis (Rudy and O'Reilly, 1999, 2001; Rudy et al., 2002). In the most compelling example, we have shown (1) that if the pre-exposed context is different from the context in which immediate shock occurs, the rat will freeze when tested in the pre-exposed context but not when tested in the context in which shock actually occurs and (2) that this result depends on the hippocampus (Rudy and O'Reilly, 2001; Rudy et al., 2002).

We have now shown that three different ways of altering the hippocampus (inactivation with muscimol, excitotoxic lesion, and protein synthesis inhibition) each block the CPFE. These results are in contrast to the findings that neither excitotoxic, anterograde damage to the DH (Maren et al., 1997; Richmond et al., 1999; Rudy et al., 2002) nor inactivating the DH before conditioning impair normal fear conditioning (experiment 4). It is of some interest that the protein synthesis inhibitor anisomycin impaired the consolidation of the memory for context when it was injected into the DH after context pre-exposure (Barrientos et al., 2002). In contrast, although muscimol blocked the acquisition of the context memory when injected before exposure, it did not block the consolidation of the memory when given after exposure. This finding suggests that, once initiated, the cellular-molecular processes needed to consolidate the context memory continue, although the synaptic transmission in the hippocampus has been greatly reduced.

We made two points in the introduction to experiment 5: (1) for context pre-exposure to enhance conditioning, the memory representation of the pre-exposed context has to be activated before the immediate shock; and (2) this retrieval process requires a hippocampus-dependent conjunctive representation. Consequently, conjunctive theory predicts that the hippocampus must be functional at the time of immediate shock (when the memory has to be retrieved). The results of experiment 5 are consistent with this prediction, because inactivating the $\mathrm{DH}$ before the immediate-shock phase blocked the CPFE. The finding that inactivating the $\mathrm{DH}$ before the fear test reduced the CPFE (experiment 6) also supports the general idea that the hippocampus is essential to retrieve a conjunctive representation of the context.

Injecting muscimol into the $\mathrm{DH}$ before conditioning did not impair contextual fear conditioning (experiment 4). This result is somewhat puzzling, because other pharmacological agents injected into the hippocampus before conditioning impair the acquisition of contextual fear conditioning. For example, Young et al. (1994) reported that injecting the NMDA receptor antagonist DL-2-amino-5-phosphonovalerate into the DH impaired the acquisition of contextual fear (also see Bast et al., 2003). Others have reported a similar impairment with scopolamine, a cholinergic antagonist (Gale et al., 2001; Wallenstein and Vago, 2001). Rudy et al. (2004) have suggested that these discrepant results can be explained as reflecting two different roles played by the hippocampus in contextual fear conditioning: (1) it stores the conjunctive representation of the context, and (2) its output inhibits conditioning to the independent features that make up the context. 
According to Rudy et al. (2004), blocking neural transmission in the hippocampus with muscimol is assumed to interfere with both functions. Thus, although the hippocampus does not store the conjunctive representation, it also does not prevent conditioning to context features, so conditioning is still robust. In contrast, an NMDA antagonist could selectively impair the storage function (Steele and Morris, 1999; Day et al., 2003; Reidel et al., 2003 ) without interfering with the inhibitory function, with a net result of reduced contextual fear conditioning. Rudy et al. (2004) did not specify just how the output of the hippocampus prevents conditioning to the context features. So at this point, it is just a hypothesis about a function that the hippocampus contributes to fear conditioning. Nevertheless, it makes a testable prediction: that an AMPA receptor antagonist such as 6-cyano-7-nitroquinoxaline, like muscimol, will not impair contextual fear conditioning. This is because blocking AMPA receptors should greatly reduce synaptic transmission through the hippocampus (Reidel et al., 2003) and thus block both the storage and inhibitory functions of the hippocampus, thereby allowing conditioning to context features.

In conclusion, by using muscimol to temporarily inactivate the $\mathrm{DH}$ at three different phases of the procedure that produces this CPFE, we show that the hippocampus is necessary for (1) acquisition of the context memory, (2) retrieval of this memory at the time of immediate shock, and (3) retrieval of the contextshock memory at the time of testing. In contrast, inactivating the $\mathrm{DH}$ before a standard contextual shock experience had no effect on contextual fear conditioning. These results support the hypothesis that two processes can support contextual fear conditioning: (1) conditioning to the conjunctive representation of the context, which depends on the hippocampus, and (2) conditioning to the independent context features, which does not.

\section{References}

Anagnostaras SG, Maren S, Fanselow MS (1999) Temporally graded retrograde amnesia of contextual fear after hippocampal damage in rats: within-subjects examination. J Neurosci 19:1106-1114.

Barrientos RM, O’Reilly RC, Rudy JW (2002) Memory for context is impaired by injecting anisomycin into dorsal hippocampus following context exploration. Behav Brain Res 134:291-298.

Bast T, Zhang WN, Feldon J (2003) Dorsal hippocampus and classical fear conditioning to tone and context in rats. Effects of local NMDA receptor blockade and stimulation. Hippocampus 13:657-675.

Bellgowan PSF, Helmstetter FJ (1995) Effects of muscimol applied to the dorsal hippocampus on the acquisition and expression of cued versus contextual fear conditioning. Soc Neurosci Abstr 21:1219.

Corcoran KA, Maren S (2001) Hippocampal inactivation disrupts contextual retrieval of fear memory after extinction. J Neurosci 21:1720-1728.

Day M, Langston R, Morris RG (2003) Glutamate-receptor-mediated encoding and retrieval of paired associate learning. Nature 44:205-209.

Edeline J-M, Hars B, Hennevin E, Cotillon N (2002) Muscimol diffusion after intercerebral microinjections: a reevaluation based on electrophysiological and autoradiographic quantifications. Neurobiol Learn Mem 7:100-124.

Fanselow MS (1990) Factors governing one trial contextual conditioning. Anim Learn Behav 18:264-270.

Fanselow MS (1999) Learning theory and neuropsychology: configuring their disparate elements in the hippocampus. J Exp Psychol Anim Behav Process 25:275-283.

Fanselow MS (2000) Contextual fear, gestalt memories, and the hippocampus. Behav Brain Res 110:73-81.

Frankland PW, Cestari V, Filipkowski RK, McDonald R, Silva A (1998) The dorsal hippocampus is essential for context discrimination, but not for contextual conditioning. Behav Neurosci 112:863-875.

Gale GD, Anagnostaras SG, Fanselow MS (2001) Cholinergic modulation of Pavlovian fear conditioning: effects of intrahippocampal scopolamine infusion. Hippocampus 11:371-376.
Gewirtz JC, McNish KA, Davis M (2000) Is the hippocampus necessary for contextual fear conditioning? Behav Brain Res 110:83-95.

Holt W, Maren S (1999a) Muscimol inactivation of the dorsal hippocampus impairs contextual retrieval. J Neurosci 19:9054-9062.

Holt W, Maren S (1999b) The ventral subiculum is required for learning auditory but not contextual fear in rats. Soc Neurosci Abstr 25:1616.

Kiernan MJ, Westbrook RF (1993) Effects of exposure to a to-be-shocked environment upon the rat's freezing response: evidence for facilitation, latent inhibition, and perceptual learning. Q J Exp Psychol B 46:271-288.

Kim JJ, Fanselow M (1992) Modality-specific retrograde amnesia of fear. Science 256:675-677.

Maren S, Fanselow MS (1998) Electrolytic lesions of the fimbria/fornix, dorsal hippocampus, or entorhinal cortex produce anterograde deficits in contextual fear conditioning in rats. Neurobiol Learn Mem 67:142-149.

Maren S, Aharonov G, Fanselow MS (1997) Neurotoxic lesions of the dorsal hippocampus and Pavlovian fear conditioning. Behav Brain Res 88:261-274.

Marr D (1971) Simple memory: a theory for archicortex. Philos Trans R Soc Lond B Biol Sci 262:23-81.

Martin JH (1991) Autoradiographic estimation of the extent of reversible inactivation produced by microinjections of lidocaine and muscimol in the rat. Neurosci Lett 127:160-164.

Martin JH, Ghez C (1999) Pharmacological inactivation in the central control of movement. J Neurosci Methods 86:145-159.

McNaughton BL, Morris RGM (1987) Hippocampal synaptic enhancement and information storage within a distributed memory system. Trends Neurosci 10:408-415.

O’Reilly RC, Rudy JW (2001) Conjunctive representations in learning and memory: principles of cortical and hippocampal function. Psychol Rev 108:311-345.

Overton DA (1991) Historical context of state-dependent learning and discriminative drug effects. Behav Pharmacol 2:253-264.

Phillips RG, LeDoux JE (1992) Differential contribution of amygdala and hippocampus to cued and contextual fear conditioning. Behav Neurosci 106:274-285.

Phillips RG, LeDoux JE (1994) Lesions of the dorsal hippocampal formation interfere with background but not foreground contextual fear conditioning. Learn Mem 1:34-44.

Reidel G, Platt B, Micheau J (2003) Glutamate receptor function in learning and memory. Behav Brain Res 140:1-47.

Richmond MA, Yee B, Pouzet B, Veenman L, Rawlins JNP, Felden J, Bannerman DM (1999) Dissociating context and space within the hippocampus: effects of complete, dorsal, and ventral excitotoxic hippocampal lesions on conditioned freezing and spatial learning. Behav Neurosci 113:1189-1203.

Rudy JW, O’Reilly RC (1999) Contextual fear conditioning, conjunctive representations, pattern completion, and the hippocampus. Behav Neurosci 113:867-880.

Rudy JW, O'Reilly RC (2001) Conjunctive representations, the hippocampus, and contextual fear conditioning. Cogn Affect Behav Neurosci 1:66-82.

Rudy JW, Barrientos RM, O'Reilly RC (2002) Hippocampal formation supports conditioning to memory of a context. Behav Neurosci 116:530-538.

Rudy JW, Huff N, Matus-Amat P (2004) Understanding contextual fear conditioning: insights from a two process model. Neurosci Biobehav Rev, in press.

Sanders MJ, Wiltgen BJ, Fanselow MS (2003) The place of the hippocampus in fear conditioning. Eur J Pharmacol 463:217-223.

Selden NR, Everitt BJ, Jarrard LE, Robbins TW (1991) Complementary roles for the amygdala and hippocampus in aversive conditioning to explicit and contextual cues. Neuroscience 42:335-350.

Spear NE (1973) Retrieval of memory in animals. Psychol Rev 80:163-194.

Steele RJ, Morris RG (1999) Delay-dependent impairment of a matchingto-place task with chronic and intrahippocampal infusion of the NMDAantagonist D-AP5. Hippocampus 9:118-136.

Wallenstein GV, Vago DR (2001) Intrahippocampal scopolamine impairs both acquisition and consolidation of contextual fear conditioning. Neurobiol Learn Mem 75:245-252.

Watkins LR, Wiertelak EP, Maier SF (1992) Delta opiate receptors mediated tail-shock induced antinociception at supraspinal levels. Brain Res 582:10-21.

Young SL, Bohenek DL, Fanselow MS (1994) NMDA processes mediate anterograde amnesia of contextual fear conditioning induced by hippocampal damage: immunization against amnesia by context preexposure. Behav Neurosci 108:19-29. 